\title{
El impacto ambiental de los residuos de promoción electoral en Ecuador: caso de estudio de las lonas publicitarias
}

\section{Environmental impact of electoral campaigns waste in Ecuador: advertising banners case}

\author{
Hellen Arichábala-Martínez $\mathbb{0}^{1 *}$
}

*Autor de correspondencia: hellenarichabala@gmail.com

Recibido: 5 de abril de 2018 - Aceptado: 23 de noviembre de 2018

1. Universidad de Especialidades Espíritu Santo, Guayaquil, Ecuador

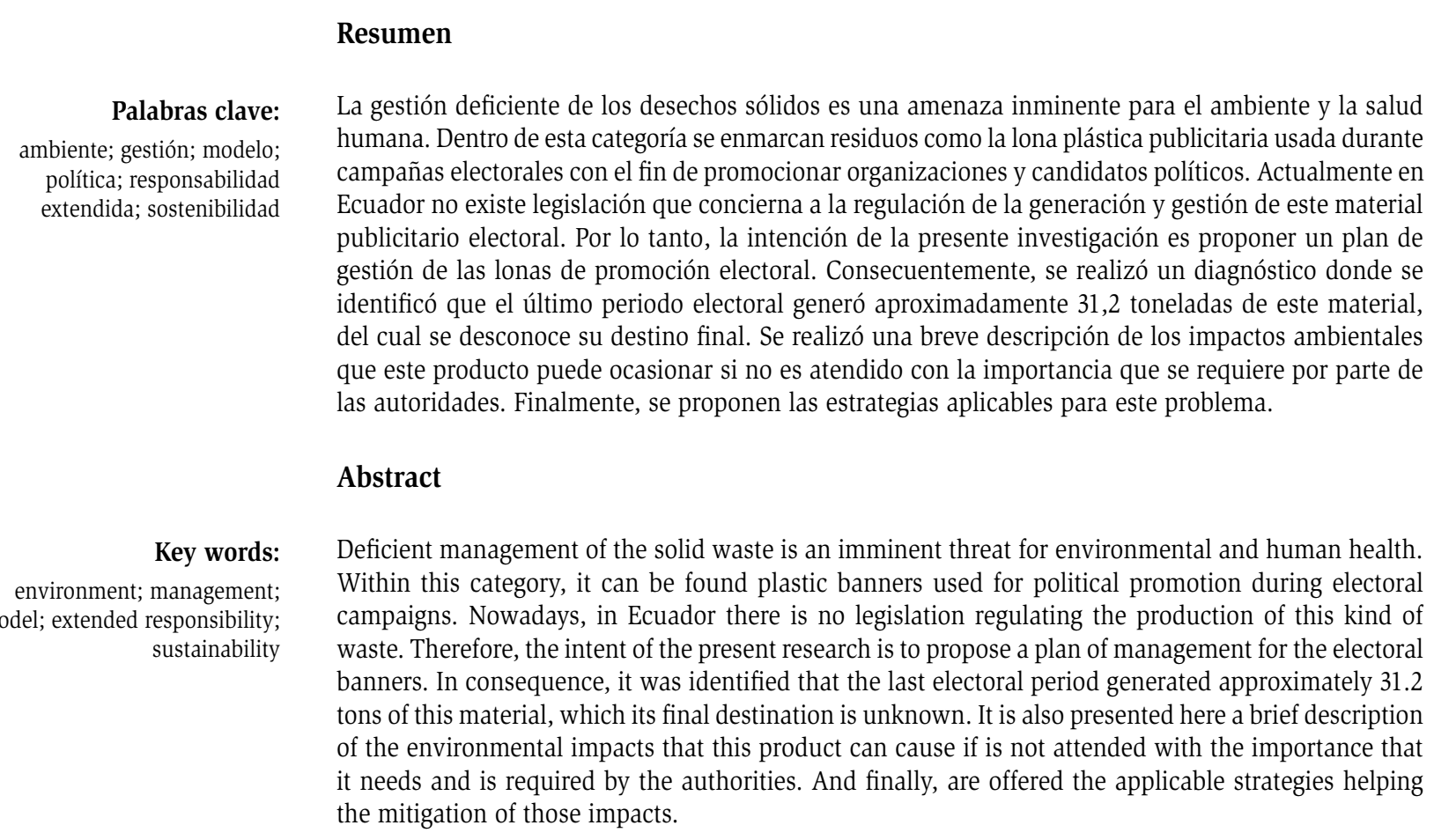

\section{Resumen}

La gestión deficiente de los desechos sólidos es una amenaza inminente para el ambiente y la salud humana. Dentro de esta categoría se enmarcan residuos como la lona plástica publicitaria usada durante campañas electorales con el fin de promocionar organizaciones y candidatos políticos. Actualmente en Ecuador no existe legislación que concierna a la regulación de la generación y gestión de este material publicitario electoral. Por lo tanto, la intención de la presente investigación es proponer un plan de gestión de las lonas de promoción electoral. Consecuentemente, se realizó un diagnóstico donde se identificó que el último periodo electoral generó aproximadamente 31,2 toneladas de este material, del cual se desconoce su destino final. Se realizó una breve descripción de los impactos ambientales que este producto puede ocasionar si no es atendido con la importancia que se requiere por parte de las autoridades. Finalmente, se proponen las estrategias aplicables para este problema.

\section{Abstract}

Deficient management of the solid waste is an imminent threat for environmental and human health. Within this category, it can be found plastic banners used for political promotion during electoral campaigns. Nowadays, in Ecuador there is no legislation regulating the production of this kind of waste. Therefore, the intent of the present research is to propose a plan of management for the electoral banners. In consequence, it was identified that the last electoral period generated approximately 31.2 tons of this material, which its final destination is unknown. It is also presented here a brief description it needs and is required by the authorities. And finally, are offered the applicable strategies helping the mitigation of those impacts. 


\section{El manejo de los desechos sólidos en Ecuador}

El manejo eficiente de los desechos sólidos continúa siendo un reto significativo para los países en vías de desarrollo. Algunos autores lo atribuyen principalmente a causas como la creciente generación de residuos, a la carga que representa en el presupuesto de los gobiernos, a la falta de reconocimiento de los procesos de gestión de residuos y sistema de manipulación, entre otros.

La existencia de nuevas alternativas de gestión de los residuos sólidos urbanos, cada vez más frecuentes, se centra en la reducción de la generación de desechos, principalmente mediante la inserción de productos ecológicos en el mercado comercial y las actividades diarias. Sin embargo, pese a la formulación de medidas preventivas y reductivas, la producción de desechos es inevitable. Razón por la cual el aprovechamiento, reciclaje y/o recuperación se reconocen como etapas viables para la gestión de los residuos. Finalmente, en caso de no ser posible el uso de las alternativas antes mencionadas, se considera como última opción la disposición final o eliminación bajo técnicas no invasivas (Ministerio de Ambiente Colombia, 2005).

Hasta el momento no existen estudios que cuantifiquen el impacto ambiental o valoren el daño ocasionado por los residuos publicitarios asociados a la demanda en procesos de promoción electoral, especialmente en países democráticos. En Ecuador, por su parte, no es distinta la problemática de una gestión deficiente de los residuos generados durante las campañas políticas. En este sentido, comprendiendo que la solución a la problemática planteada debe estar alineada a la realidad ambiental actual del país, se establece como objetivo general proponer un plan de gestión enmarcado dentro de una política ambiental para los desechos generados durante los períodos de campaña electoral, estableciendo estrategias que deberán ser cumplidas por los partidos, asociaciones políticas, frentes y otros, durante y después de esos episodios. Por ello, se planteó estimar las cantidades de lona usada en las campañas publicitarias electorales del 2017 y mencionar los principales problemas que generan los residuos de lonas publicitarias desechadas tras culminar las elecciones presidenciales. Todo ello con el interés de coadyuvar al control de la contaminación y disminuir la cantidad de este tipo de plástico destinado al relleno sanitario.

\section{Las campañas electorales en el Ecuador}

La historia política de Ecuador está caracterizada por los cambios electorales y campañas políticas; durante el 167 periodo electoral, los diferentes partidos políticos adoptan tácticas para obtener el voto de los sectores titubeantes y afianzar su lugar en la tabla de posiciones. Por lo tanto, el uso de la promoción es clave (Reyes, 2017). Según el Consejo Nacional Electoral (CNE), se clasifica como promoción el uso de: la radio, la televisión, la prensa y las vallas publicitarias (Consejo Nacional Electoral CNE, 2017). Además de estas estrategias, los candidatos a la presidencia y/o alcaldía (considerando las dignidades políticas más altas) utilizan como medio de fomento popular el acercamiento puerta a puerta con la ciudadanía, recorrido por los sectores más vulnerables y difusión de afiches, camisetas y grandes banners que hacen referencia al partido de su correspondencia.

Por otra parte, el gasto en el que se incurre por dicha promoción es elevado, para el proceso electoral del año 2017, la normativa indicó que en primera vuelta, el presupuesto máximo de las elecciones presidenciales se estableció en \$ 1922504,70 , donde el $30 \%$ del valor total corresponde al gasto por promoción equivalente a 576 751,41 dólares (Ecuavisa, 2016). En lo que respecta a la segunda vuelta, se aprobó un incremento del fondo de promoción electoral a \$ 769 001,88 sobre el total (El Comercio, 2017).

Las campañas normalmente tienen un tiempo de duración de 45 días donde pueden hacer uso del fondo estipulado para fines estrictamente políticos y a través de los medios que el CNE considera aptos para la promoción. De acuerdo al Reporte de Proveedores y Fondo de Promoción Electoral (Consejo Nacional Electoral CNE, 2017), el 11,21 $\%$ de los proveedores registrados corresponde a vallas publicitarias, el 13,43 \% a prensa, el 14,37 \% a televisión y el $60,98 \%$ corresponde a radio. En la última campaña electoral realizada en Ecuador, en el año 2017, el fondo de promoción total utilizado en la primera vuelta electoral se dividió de la siguiente manera: $\$ 935176,50$ para vallas publicitarias, \$ 1629748,14 para prensa, \$ 9411 397,95 para radio y $\$ 11488$ 675,61 para televisión.

\section{Residuos de campaña}

Los residuos de campaña son todos aquellos materiales e insumos utilizados con fines publicitarios de promoción electoral que, una vez cumplen su uso (fin del periodo de campaña), son desechados y no se vuelven a utilizar. En México se estima que existe un incremento de la producción del material publicitario de tres veces la cantidad usual por cada campaña electoral (Excélsior, 2018).

Diversos líderes políticos coinciden en que no hacer uso de las herramientas visuales de difusión les representaría 
una desventaja frente a sus oponentes. En otras palabras, la promoción electoral que se da por medio de vallas se la considera "un mal necesario" puesto que otorga posicionamiento social al candidato (Sandoval, 2012). Sin embargo, esta opción de promoción electoral genera un desecho no clasificado en la normativa ambiental ecuatoriana: gallardetes de polietileno con un arte impreso sobre ellos (Acuerdo Ministerial No. 142 con Registro Oficial No. 856 del Ministerio del Ambiente; Código Orgánico del Ambiente, 2017).

El material impreso en lonas es uno de los principales canales a considerar para la promoción, utilizado como un mecanismo para ganar adeptos para el partido y el candidato. El proceso de elaboración de las lonas producidas por la industria gráfica conlleva un impacto ambiental implícito. En primera instancia, en la etapa de secado de las lonas publicitarias se da una emisión de solventes volátiles con una gran proporción de componentes clorados. Cabe mencionar que el principal constituyente de la lona es el PVC, un material no biodegradable con propiedades impermeables, persistentes y altamente tóxicas (Arichábala et al., 2014). Una vez más, la utilización de gas cloro y compuestos organoclorados durante la producción de PVC exalta su peligrosidad. Asimismo, este material es muy inflamable al contacto con el fuego, se emanan metales pesados, dioxinas y furanos (compuestos cancerígenos), y cloruro de hidrógeno que reacciona con la humedad y forma ácido clorhídrico (Greenpeace, 2001).

Otra consideración que agrava el problema de la generación de este tipo de insumo publicitario y posterior desecho es su baja posibilidad de reciclaje. Las vallas son categorizadas como un material de característica reciclable baja por la complejidad de los aditivos que las componen. De igual manera, los intentos de reciclaje no son rentables puesto que los costos son más elevados que la materia prima (Organización de Usuarios y Trabajadores de la Química del Cloro (AMICLOR), 2017). En México las campañas electorales intermedias del año 2013 generaron 400 toneladas de residuos plásticos que, de acuerdo a la Universidad Popular Autónoma del Estado de Puebla, tardarán por lo menos 20 años para degradarse (Villafranco, 2017). En Ecuador, por su parte, la Empresa Pública Metropolitana de Aseo operó un plan en el Distrito Metropolitano de Quito para la recolección de la "basura publicitaria electoral" correspondiente al proceso electoral del 19 de febrero de 2017 y las manifestaciones consecuentes, de lo que resultó en un total de 660 toneladas de residuos mixtos recogidos (Corresponsables, 2017). Acciones similares no se han desarrollado en la ciudad de Guayaquil donde se asegura que el manejo de los residuos continúa siendo un problema. Según las cifras oficiales, la ciudad originó en el año 2015 un total de 3963 toneladas de desechos sólidos por día (Diario El Telégrafo, 2016).

Por otra parte, en el año 2014 se realizó el estudio "Propuesta de gestión sostenible de los residuos plásticos producidos por la industria gráfica en la ciudad de Guayaquil" donde se planteó un modelo sostenible de gestión de residuos por medio de la reutilización de los desechos plásticos generados por la industria gráfica, con el fin de reducir la contaminación y los problemas de drenaje de lixiviados en el relleno sanitario Las Iguanas; esta iniciativa generó paralelamente una fuente de trabajo (Arichábala et al., 2014). Cabe mencionar que la propuesta no solo estaba enfocada en desechos de publicidad electoral, sino también en todos los desechos plásticos de vallas publicitarias demandadas en la urbe. Consiguientemente, se exhorta a los Gobiernos Autónomos Descentralizados para que consideren dicho modelo de gestión como un lineamiento base para el manejo de los desechos de lonas de publicidades comunes.

\section{Bases teóricas de las políticas}

Las políticas proveen una respuesta o solución a definidas situaciones que han sido reconocidas como preocupaciones relevantes en algunos entornos delimitados de realidad. Dicho de otra forma, "las políticas públicas corresponden a soluciones específicas de cómo manejar los asuntos públicos" (Subsecretaría de Desarrollo Regional y Administrativo, SUBDERE, 2009). El Sistema Nacional Descentralizado de Planificación Participativa creado en la Constitución del 2008 otorgó la vinculación con el sector público por medio del Nacional para el Buen Vivir (PNBV). Con el PNBV se abre paso para la creación de nuevas políticas a fin de garantizar el desarrollo social del país (Secretaría Nacional de planificación y desarrollo, SENPLADES, 2011). Además, Como referente internacional, el artículo 286 del Código Federal de Instituciones y Procedimientos Electorales de México establece que los políticos deberán usar para sus propagandas impresas materiales "que no dañen el medio ambiente, preferiblemente reciclables y de fácil degradación natural”. Situación que en el contexto de nuestra área de estudio carece de disposiciones similares hasta el momento.

Dicho esto, la necesidad de una política es clave para una gestión correcta de los desechos electorales en Ecuador puesto que en la actualidad no existe norma, ley o reglamentación específica que dictamine las responsabilidades y/o obligaciones de las organizaciones políticas como generadores de dichos residuos. Existen 
unas normativas que regulan todo el proceso electoral (la Ley Orgánica Electoral, código de la Democracia, el Código del Ambiente de 2011 y, el Reglamento de Promoción Electora de 2015), pero estas no consideran los desechos generados por la industria publicitaria dentro de las regulaciones vigentes en el país, lo cual determina un vacío legal sobre esta temática.

\section{Determinación de las cantidades de residuo generado}

Mediante la revisión literaria de las fuentes oficiales de datos como el CNE se determinó la cantidad del gasto económico destinado a vallas publicitarias durante las elecciones presidenciales 2017. Esto se obtuvo del Reporte de Proveedores y Fondo de Promoción Electoral. Adicionalmente, las empresas publicitarias e imprentas habilitadas para ofrecer sus servicios a las organizaciones políticas debieron pasar por un proceso de calificación. Por esto son limitadas en cantidad y se encuentran distribuidas en todo el país, razón por la cual se optó por el método de entrevista telefónica. Se procedió a realizar la entrevista telefónica a 15 de los 22 proveedores registrados seleccionados al azar, esta es una muestra significativa con $95 \%$ de nivel de confianza y $15 \%$ de margen de error.

En adición a las entrevistas a los proveedores calificados de vallas publicitarias, se intentó tener un acercamiento por medio de encuestas a representantes de los partidos políticos, con la finalidad de obtener datos más precisos sobre la cantidad final producida, pero no se obtuvo respuesta.

La disponibilidad de información acerca de la cantidad de generación y gestión de los residuos producidos por la promoción de los partidos políticos durante el periodo de campaña es escasa, deficiente, dispersa y en su mayoría inexistente. Por consiguiente, el levantamiento de un diagnóstico es limitado. A pesar de las dificultades, se puede indicar que: se estimó la cantidad por medio del cálculo de la suma del gasto total destinado a vallas publicitarias de todas las organizaciones políticas dividido por el precio medio del metro cuadrado vendido por parte de la muestra de 15 proveedores autorizados y registrados.

De acuerdo a las entrevistas telefónicas, el precio medio de venta de una lona de $3 \times 2 \mathrm{~m}$, fue de $\$ 90$. Ahora bien, partiendo de un total de $\$ 935$ 176,50 destinados para lonas publicitarias, se estimó un total de $62345,10 \mathrm{~m}^{2}$ equivalentes a $31172 \mathrm{Kg}$. Esto equivale a 31 toneladas únicamente de lona con probabilidad de ser desechadas 169 como basura común.

\section{Buenas prácticas propuestas}

Se plantean las siguientes estrategias como buenas prácticas en la producción, uso y gestión de material electoral publicitario hecho en lona:

Estrategia 1. Fabricación y contratación de los productos plásticos publicitarios.

a.Los proveedores son responsables del uso de materiales ecológicamente más viables.

b.Las entidades políticas deberán exigir por parte de los proveedores las certificaciones e indicadores de cumplimiento de los programas establecidos en esta política.

c. Los proveedores deberán tener un control del peso, dimensiones y tipo de materiales usados en un registro de venta.

d.Los proveedores deberán gestionar sus desechos de producción con gestores autorizados dependiendo la naturaleza del desecho según la normativa legal aplicable.

e. Las entidades políticas tienen el deber de cuidar el ambiente optando responsablemente por otras fuentes de promoción con el fin de reducir el uso de lonas de PVC (vallas publicitarias).

Estrategia 2. Recolección. Las entidades políticas tienen un máximo de 7 días tras culminado el uso de la lona para recolectarlas en los puntos de colocación.

Estrategia 3. Almacenamiento y clasificación. Las entidades políticas tienen el deber de una vez recolectadas las lonas, almacenarlas de manera temporal en su centro de acopio donde serán clasificadas y redistribuidas para su reciclaje y/o aprovechamiento.

Estrategia 4. Reciclaje. Las entidades políticas tienen el deber de otorgar un segundo uso sostenible a las lonas recolectadas. Así mismo, deberán realizar acciones compensatorias del impacto ambiental generado que fomente la participación pública en temas ambientales.

Estrategia 5. Tratamiento. Únicamente en casos extraordinarios, de ser imposible el aprovechamiento o 
reciclaje de la lona, se deberá buscar un tipo de tratamiento controlado para la misma.

a.Incineración. Solamente podrá efectuarse si el gestor cumple con los parámetros mínimos de destrucción total de los componentes de la lona de PVC y posee los componentes mínimos necesarios de prácticas sostenibles con el fin de evitar otras fuentes de contaminación, entre las cuales figuran: depurador de gases y planta de tratamiento de aguas residuales.

b. Disposición final. En caso que la incineración controlada presente un riesgo para la calidad ambiental, debido a falta de tecnologías de incineración eficientes en el país, podrá darse una disposición final en confinamientos especializados (conocidos como celdas de confinamiento). De esta manera, si en un futuro se llegara a contar con la tecnología que garantice su aprovechamiento 0 eliminación (por ejemplo: técnicas más avanzadas de reciclaje o incineradores más limpios) se podría retirarlas de su confinamiento para brindarles un tratamiento sostenible.

Estas estrategias son un eje fundamental para la política, las mismas que atajan los objetivos correspondientes a cada aspecto ambiental. En cuanto a su formulación legislativa, es decir, en forma de artículos o cláusulas legales, es de competencia del organismo del ramo encargado al enunciado de las leyes. Por lo tanto, la presente es una propuesta netamente enmarcada en parámetros de las ciencias ambientales que deberán ser estructurados de forma tal para que cumplan con las cualidades de órgano legal al momento de su expedición.

Es necesario mencionar que las implementaciones de estas estrategias dependen de una política pública. Si bien es cierto que su valorización económica está directamente relacionada con el presupuesto de campaña de cada partido político, es un gasto necesario que debería ser provisionado con anticipación por cada organización política como parte inamovible de su proceso de campaña electoral.

\section{Conclusiones}

Se concluye que en el proceso de elección presidencial llevado a cabo en el año 2017 en Ecuador: se generaron 31,2 toneladas de desechos correspondientes únicamente a lonas publicitarias, la gestión de los residuos de campaña no fue un proceso regularizado, no existe un levantamiento estadístico de la tipología y cantidad de generación y gestión de los desechos urbanos en general, los partidos políticos tienen un control deficiente de la cantidad gestionada de los desechos generados en campaña y, por último, no existen estadísticas del tipo de impresión, tamaño, calidad de impresión, unidades impresas por cada partido político.

Por las anteriores razones, es primordial realizar un plan de gestión que esté respaldado por una política pública para prevenir el potencial daño ambiental y regular la generación, uso y gestión de los desechos producidos por medio de las estrategias planteadas de buenas prácticas de antes, durante y después del uso de dicho material, pero que también posea el carácter legal que condiciona a las entidades políticas a garantizar el éxito del plan de acción.

\section{Agradecimientos}

A mis padres y a Ivanna Terán por la retroalimentación del trabajo.

\section{Referencias}

Arichábala Martínez, H. 2014. Propuesta de gestión sostenible de los residuos plásticos producidos por la industria gráfica y publicitaria en la ciudad de Guayaquil. Premios Odebrecht, Quito.

Código Orgánico del Ambiente. 2017. Registro Oficial Suplemento No. 983 del 12 de abril de 2017.

Consejo Nacional Electoral (CNE). 2016. Calendario Electoral 2016-2017. http://www.ecuavisa.com/sites/default/files/ calendario_electoral_2016-2017.pdf. Consultado: 8 de enero 2018.

Consejo Nacional Electoral (CNE). 2017. Reportes: Proveedores y Fondo de Promoción Electoral. Promoción Electoral Elecciones Generales 2017. https://app05.cne. gob.ec/VeeduriaPromocionElectoral/frmVeeduriaPrincipal. aspx?pg = 1. Consultado: 28 de marzo de 2018.

Corresponsables. 2017. 660 toneladas de basura se recolectó en "Elecciones 2017". Disponible bajo la siguiente dirección: http://ecuador.corresponsables.com/actualidad/660toneladas-de-basura-se-recolecto-en-elecciones-2017. Consultado: 15 de marzo de 2018.

Diario El Telégrafo. 2016. El manejo de la basura sigue siendo un problema. http://www.eltelegrafo.com.ec/noticias/ guayaquil/10/el-manejo-de-la-basura-sigue-siendo-unproblema. Consultado: 03 de mayo de 2016. 
Excelsior. 2018. Las 3 delegaciones que generan mayor cantidad de basura en la CDMX. https://www.excelsior. com.mx/comunidad/las-3-delegaciones-que-generanmayor-cantidad-de-basura-en-la-cdmx/1260958. Consultado 26 de agosto de 2018.

Ecuavisa, 2016. CNE establece presupuesto para elecciones del 2017. http://www.ecuavisa.com/articulo/noticias/ politica/217996-cne-establece-presupuesto-eleccionesdel-2017. Consultado: 03 de mayo de 2017.

El Comercio. 2017. CNE incrementó el fondo de promoción electoral para la segunda vuelta. http://www.elcomercio.com/ actualidad/cne-incremento-fondo-promocion-elecciones. html. Consultado: Consultado: 8 de enero 2018.

Greenpeace. 2001. Hacia un futuro libre de PVC. España. http://www.greenpeace.org/espana/es/reports/hacia-unfuturo-libre-de-pvc/. Consultado: 28 de marzo 2017.

Ley Orgánica Electoral, Código de la Democracia. Registro Oficial Suplemento No. 578 del 27 de abril de 2009 (última reforma el 21 de enero de 2011).

Ministerio de Ambiente Colombia. 2005. Política Ambiental para la Gestión Integral de los Residuos o Desechos Peligrosos. http://www.un.org/esa/dsd/dsd_aofw_ni/ni_pdfs/ NationalReports/colombia/Gestion_de_ResiduosWaste_management.pdf .Consultado: 15 de marzo 2018.

Organización de Usuarios y Trabajadores de la Química del Cloro (AMICLOR). 2017. ¿Dónde está la toxicidad del PVC?http://revistainterforum.com/
espanol/articulos/102103Naturalmente_toxicidad-vc. html iframe $=$ true\& width $=95 \%$ \&height $=95 \%$. Consultado: 4 de marzo 2018.

Reglamento de Promoción Electoral. Registro Oficial No. 671 del 23 de diciembre de 2015 (última reforma el 17 de marzo de 2017).

Reyes, N. 2017. Análisis político del arranque de campaña electoral en el Ecuador. La Junta. http://lajunta.ec/2017/01/23/ analisis-politico-del-arranque-campana-electoral-ecuador/. Consultado: 28 de marzo 2017.

Sandoval, F. 2012. La propaganda electoral, un "mal necesario". http://www.animalpolitico.com/2012/02/lapropaganda-electoral-un-mal-necesario/ Consultado: 28 febrero de 2018.

Secretaría Nacional de planificación y desarrollo (SENPLADES). 2011. Guía para la Formulación de Políticas Públicas Sectoriales. Subsecretaria de Planificación Nacional, Territorial y Políticas Públicas. SENPLADES, Quito.

Subsecretaría de Desarrollo Regional y Administrativo (SUBDERE). 2009. Guía Metodológica para la Formulación de Políticas Públicas Regionales. Gobierno de Chile. Ministerio del Interior. Santiago de Chile.

Villafranco, G. 2017. Propaganda política, icomunicación efectiva o dinero tirado a la basura? Revista Forbes México. https://www.forbes.com.mx/propaganda-politicacomunicacion-efectiva-o-dinero-tirado-a-la-basura/ Consultado: 25 febrero de 2018.

Citar como: Arichábala-Martínez, H. 2018 El impacto ambiental de los residuos de promoción electoral en Ecuador: caso de estudio de las lonas publicitarias. Intropica 13(2): 166-171 DOI: http://dx.doi.org/10.21676/23897864.2640. 


\section{GUÍA PARA AUTORES}

INTROPICA es una revista científica de publicación semestral, arbitrada por evaluadores nacionales e internacionales que provee un espacio para la publicación de contribuciones originales e inéditas que estudien e interpreten de manera integrada los ecosistemas tropicales así como los problemas ambientales derivados de la intervención antropogénica sobre el territorio.

La revista INTROPICA está disponible en versión digital con acceso libre (http://intropica.unimagdalena.edu.co) y en versión impresa (ISSN 1794-161X), la cual se distribuye en bibliotecas especializadas de instituciones académicas, gubernamentales y no gubernamentales.

\section{Envío:}

Los manuscritos pueden ingresar al proceso editorial a través de la plataforma Open Journal System de la revista (http:// intropica.unimagdalena.edu.co) o a través del correo electrónico revistaintropica@unimagdalena.edu.co. La recepción de manuscritos es permanente. En el envío se deberán adjuntar las figuras en archivos independientes y una carta de aprobación de todos los autores, certificando su originalidad y que no ha sido remitida ni se ha publicado en otra revista. En la carta también deberá indicarse el tipo de contribución (artículo, artículo de revisión, artículo de reflexión o nota científica) y dos posibles evaluadores con sus respectivos datos de contacto. Aunque todas las contribuciones estarán sujetas a revisión previa, la responsabilidad por el contenido de las mismas recae sobre los autores y no sobre el editor, el Comité Editorial o la Universidad del Magdalena. El formato para elaborar esta carta remisoria puede descargarse del sitio de Internet de la revista.

\section{Tipos de contribuciones:}

a) Artículo de investigación científica y tecnológica. Corresponden a resultados completos de estudios realizados sobre las temáticas que aborda la revista. Los manuscritos tendrán un máximo de 30 páginas, incluyendo las tablas y las figuras. El artículo de investigación deberá seguir el siguiente orden: Título, Autor (es), Dirección postal del (de los) autor (es), Resumen, Palabras claves, Abstract, Key words, Introducción, Materiales y Métodos, Resultados, Discusión, Conclusiones (opcional), Agradecimientos (opcional), Bibliografía, Tabla/s con su/s correspondiente/s leyenda/s, Lista de las leyendas de las figuras. Las figuras deben ser enviadas en archivos independientes en formato gráfico (tif, gif, eps).

b) Artículo de revisión. Se refieren a trabajos de revisión crítica que compilan y resuman adecuadamente el grado de avance o del conocimiento y señalan líneas de investigación a seguir. La extensión máxima del manuscrito será de cuarenta páginas, incluyendo tablas y figuras. Deben contener al menos cincuenta referencias bibliográficas. El artículo de revisión puede escribirse en un estilo libre, sin embargo, deberá incluir resumen, palabras clave, abstract, key words, agradecimientos (opcional) y bibliografía.

c) Artículo de reflexión. Presenta la perspectiva del autor, fundamentada en literatura científica, como aporte al conocimiento de los temas propios de la revista. Los manuscritos tendrán un máximo de treinta páginas, incluyendo las tablas y las figuras. Se deberá incluir resumen, palabras clave, abstract, key words, agradecimientos (opcional) y bibliografía.

d) Nota científica. Son descripciones breves de investigaciones limitadas, procedimientos experimentales, operaciones técnicas o aplicadas. En cualquier caso, tienen que ser completamente documentadas, con referencia a la literatura, e indicando los procedimientos experimentales empleados. El manuscrito puede ocupar un máximo de 10 páginas, incluyendo las tablas y las figuras. La nota científica no debe llevar subtítulos pero sí resumen, palabras clave, abstract y key words, agradecimientos (opcional) y bibliografía. 
e) Editorial. Documento escrito por el editor, un miembro del Comité Editorial o un investigador invitado sobre orientaciones en el dominio temático de la revista. Corresponde a un documento corto con pocas referencias.

\section{Procedimiento de evaluación/arbitraje de los manuscritos}

Los editores realizarán una primera evaluación del manuscrito, conceptuando la pertinencia e idoneidad del mismo y lo someterán al proceso de arbitraje doble ciego de por lo menos dos especialistas en el tema. En caso de controversia entre los evaluadores se recurrirá a un tercero. Estos especialistas actuarán como revisores del manuscrito, emitirán su concepto y podrán realizar recomendaciones o solicitar aclaraciones en un tiempo máximo de un mes. Posteriormente, las sugerencias de los editores y revisores serán comunicadas a los autores, quienes tendrán un plazo de un mes para responder dichas sugerencias. En caso contrario, el manuscrito será dado de baja y de ser remitido nuevamente se iniciará el proceso editorial desde el principio con un nuevo arbitraje. Los editores tomarán la decisión final de aceptar o no los manuscritos para su publicación. Para estas tareas los editores podrán apoyarse en el Comité Editorial, cuando así lo estimen pertinente.

Previo a la impresión final de los manuscritos aceptados para publicación, los editores enviarán las pruebas de galera al autor de correspondencia para que efectúe correcciones por errores de digitación únicamente. No se permiten mayores cambios o adiciones al manuscrito editado en esta fase.

En caso de comprobación de plagio o de violación de derechos de autor, los editores ordenarán cancelar el proceso editorial. Para mayor información consulte la guía de ética y buenas prácticas editoriales: http://revistas.unimagdalena.edu.co/index.php/intropica/about/editorialPolicies\#custom-3

\section{Formato del manuscrito:}

El texto se debe enviar como documento en formato Word o RTF con un nombre corto que identifique al manuscrito y al primer autor (e.g. Hifomicetos_López.doc). El texto debe estar interlineado a doble espacio y numeradas las líneas a lo largo del texto (incluyendo tablas), con letra Times New Roman de 12 puntos y con todos los márgenes de $2 \mathrm{~cm}$. Se aceptan manuscritos en español o en inglés. Las páginas deben numerarse consecutivamente. Las tablas se incluirán en formato tabla (no pegarlas como imagen) al final del texto del manuscrito. Las figuras no deben incluirse en el documento del manuscrito.

Para los nombres científicos y las locuciones latinas se utilizará cursiva. No incluya palabras subrayadas ni sangrías. En el caso de citar un taxón de nivel específico o inferior, se debe citar su nombre científico incluyendo el autor. Tanto los autores como los editores se guiarán por las reglas que gobiernan la nomenclatura biológica, de acuerdo a lo acordado en el "International Code of Botanical Nomenclature", en el "International Code of Nomenclature of Bacteria" y en el "International Code of Zoological Nomenclature". Se enfatiza que el autor del nombre y el año de publicación de cada taxón solo deberán aparecer una vez, la primera vez que se mencione.

1. Título. Claro, descriptivo y no demasiado largo. Se solicita en español e inglés. El título del trabajo y los títulos de las secciones principales del manuscrito debe ubicarse en posición central y en mayúsculas; los títulos de segundo rango deben ubicarse en posición central en minúsculas y los de tercer rango alineados a la izquierda. Todos los títulos y subtítulos deben ir en negrita. Adicionalmente, debe suministrarse un título abreviado de máximo ochenta caracteres incluyendo espacios.

2. Nombre(s) del (de los) autor(es). Los nombres se deben escribir en mayúsculas y minúsculas. En el caso de varios autores, el primer nombre debe ser el del autor principal. 
3. Dirección del (de los) autor(es). Se deberá anotar institución a la que pertenecen, dirección postal y teléfono de todos los autores. Se deberá agregar el correo electrónico para correspondencia.

4. Resumen y Abstract. Deberá escribirse un resumen en español y otro en inglés. En ambos casos, no deberá exceder las 300 palabras. El resumen debe contener de manera sintética los objetivos del trabajo, una descripción concisa de la parte experimental (incluyendo los tamaños de muestra), principales resultados y conclusiones generadas en el proceso de discusión de los mismos. En el resumen, no se debe incluir referencias bibliográficas y debe escribirse en un solo párrafo. El Abstract debe ser una traducción fiel del Resumen.

5. Palabras clave y key words. Después del Resumen y del Abstract, deberán incluirse en español e inglés, respectivamente, 3 a 6 palabras clave que identifiquen el manuscrito.

6. Introducción. Se debe presentar una revisión actualizada de la bibliografía pertinente al trabajo (fuentes adecuadamente referenciadas), para fundamentar la hipótesis de trabajo y mostrar el o los objetivos propuestos.

7. Materiales y métodos. Se deberá incluir, en forma clara y concisa, los principales materiales, así como también los métodos aplicados, de modo que, con esa información, otro investigador pudiese repetir la experiencia. Pueden utilizarse apartados diferentes bajo subtítulos adecuados. No debe describirse un método si ya está descrito en la bibliografía; basta con presentar la cita bibliográfica. Si utiliza un método modificado, debe señalarse claramente la modificación.

8. Resultados. Los resultados deben presentarse en forma clara y precisa, incluyendo tablas, figuras y, en caso necesario, análisis estadísticos.

9. Discusión. Los resultados deben ser contrastados con el conocimiento registrado en la literatura, destacando el aporte del artículo para el entendimiento del tema tratado. Las conclusiones que se extraigan del trabajo deberán ser consecuentes y relacionadas con los resultados expuestos. Los resultados y discusión pueden conformar una sección.

10. Agradecimientos. De ser considerado necesario por los autores, se puede incluir algún agradecimiento por contribuciones económicas, asesorías, donaciones u otro tipo de colaboración que haya hecho posible el trabajo.

11. Referencias. Se debe listar la información completa de todas las referencias bibliográficas, las cuáles serán ordenadas alfabéticamente por el apellido del primer autor de cada cita del documento. La exactitud de las referencias bibliográficas es responsabilidad de los autores. INTROPICA utiliza el sistema Harvard de citación. Es necesario tener en cuenta el siguiente estilo de escritura:

- Debe revisarse cuidadosamente el manuscrito para verificar que el deletreado de los nombres de los autores y el año sean exactamente iguales en el texto y en la lista de referencias.

- En el texto se debe referir al (los) apellido(s) del (los) autor(es) y al año de publicación. Por ejemplo, "Rodríguez (1998) encontró que.....”. "Una técnica multinomial ha sido descrita para estimar la varianza asociada con proporciones (Kent y Lane, 2004)”.

- Cuando en el texto se citan publicaciones escritas por más de dos autores, se escribirá el nombre del primer autor seguido por "et al." sin cursiva. En la Bibliografía deben mencionarse los nombres de todos los autores.

- Si hay varios trabajos de un autor(es) en un mismo año, se citará con una letra en secuencia adosada al año (ejemplo: García et al., 1998a, b, c).

- Las citas en una misma frase del texto deben ser ordenadas cronológicamente. Por ejemplo, "Los indicadores biológicos son ampliamente usados para análisis ambientales (Platt, 1964; Arias y Pérez, 1980; Klimm et al., 2000)”. 
- El nombre de las revistas científicas debe estar completo, no abreviado y en cursiva.

- El título de las referencias debe conservar el idioma original.

- Referencias concernientes a datos no publicados (doc. ined.) y "comunicaciones personales" (com. pers.) no deben ser incluidas en la lista de referencias pero si pueden ser citados en el texto.

Use el siguiente formato en la bibliografía para citar las referencias:

- Publicaciones periódicas o revistas:

Pérez, R., Condit, R., Aguilar, S., Hernández, A. y Villareal, A. 1996. Inventario de la vegetación de la isla de Coiba, Panamá: Composición Florística. Revista de Biología Tropical 44: 31-40.

Cubides-Guerrero, P.A. y Ramírez-Franco, J.H. 2014. Adsorción de Cr VI sobre residuos de café. Revista Mutis 4(2): 18-25 http://revistas.utadeo.edu.co/index.php/mutis/article/view/953/992. Consultado: 2 de enero de 2015

- Libros:

Longhurst, A. y Pauly, D. 1987. Ecology of tropical oceans. Academic Press, San Diego.

- Capítulos de libro:

Bucker, D. 1989. Histology. En: Austri, D., Editor. Methods for the Microbiological Examination of Fish and Shellfish. Wiley, New York.

- Tesis:

Gil, A. 2000. Evolución bioquímica de los endosimbiontes en insectos asociados con el maíz en el sur de México. Tesis de Doctorado, Universidad Agrícola, Mérida, Yucatán, México.

- Sitios de Internet:

Alexander, J. y M.A. Tate. 2001. Evaluando las Fuentes Electrónicas. Widener University._URL:_http://www2. widener.edu/Wolfgram-MemorialLibrary/ webevaluation/ webeval.htm. Consultado: 9 de octubre 2012.

\section{Tablas}

- Deben elaborarse en procesador de tablas de MS Word. No utilice la barra espaciadora o la tecla de tabulación.

- Las tablas deberán ser numeradas de acuerdo a su secuencia en el texto, en orden correlativo con números arábigos y llevarán una leyenda descriptiva y breve en la parte superior, que será la referencia de la tabla. Se recomienda que la leyenda sea clara e informativa, de manera que se evite consultar el texto para entender la tabla.

- Cada tabla debe presentarse en una página separada del manuscrito. Nunca deben ser incluidas en el texto, sino presentadas al final del texto del manuscrito y antes de la lista de las leyendas de las figuras.

- La información presentada en las tablas no debe aparecer repetida en las figuras.

- Los encabezados de las columnas deben ser breves, pero explicativos. No usar negrillas. Las abreviaturas estándar de las unidades de medida deben colocarse entre paréntesis.

- No deben usarse líneas verticales para separar las columnas. En lugar de ello, deje espacio extra entre ellas.

- Cualquier explicación adicional para el entendimiento de la tabla debe suministrarse como una nota de pie de tabla. 


\section{Lista de las leyendas de las figuras}

- Al final del texto del documento y en hoja aparte se hará una lista con el texto de las leyendas de las figuras.

- No deben utilizarse negrillas y se acentuarán tanto las minúsculas como las mayúsculas.

- Se recomienda que las leyendas sean informativas para que el lector no necesite consultar el texto para entender lo que muestra la figura.

- Se recomienda que las explicaciones o las descripciones que se consideran necesarias se escriban en la misma leyenda para evitar texto dentro de las figuras.

\section{Figuras}

- Las figuras (dibujos, mapas, láminas, esquemas, gráficas de computador y fotografías) deben ser enviadas en archivos independientes, en formato digital gráfico de alta calidad (tiff, bmp, jpg o gif), con una resolución mínima de 300 dpi. De ser posible, se recomienda enviar archivos gráficos de tipo vectorial.

- El nombre del archivo correspondiente a la figura será el del primer autor seguido por el número de la figura, e.g. López fig.1.jpg.

- Las figuras serán enumeradas con números arábigos de acuerdo a su secuencia de aparición en el texto.

- Las figuras deben diseñarse teniendo en cuenta el formato de la página de la revista. Su tamaño original debe permitir una reducción del 50\%, sin pérdida de claridad.

- Las figuras compuestas con varias imágenes, se enumeraran correlativamente. Ej. Figura 1a, 1b, 1c, etc. Los dibujos deben tener una escala comparativa para determinar el aumento.

- Use la misma clase de letra en todas las ilustraciones y siga el estilo de la revista.

\section{Unidades}

Las medidas se deben expresar en unidades del sistema métrico decimal; se deja un espacio entre el número y la unidad de medida y no debe ir punto después de la abreviatura $\left(15 \mathrm{~m}, 10 \%, 20^{\circ} \mathrm{C}, 7 \mathrm{~kg}\right)$. Las unidades de medidas abreviadas únicamente deben ser usadas cuando van antecedidas de números. Los valores de salinidad deben expresarse sin unidades o símbolos.

\section{Fórmulas}

- Las ecuaciones matemáticas deben escribirse como texto editable y no como imágenes.

- Dé el significado de todos los símbolos inmediatamente después de la ecuación en la que son usados por primera vez.

- Para fracciones simples use el símbolo “/” en lugar de la línea horizontal; por ejemplo: $S y / X m$ en lugar de $\frac{S y}{X m}$

- Las ecuaciones explícitamente referenciadas en el texto deben numerarse consecutivamente al lado derecho, entre paréntesis.

- El nivel de significancia estadística debe indicarse con la letra "p" minúscula y sin espacio entre el signo y el valor de significancia. Ejemplo: $\mathrm{p}<0,05$.

\section{Cifras decimales}

En lo que respecta a las cifras decimales, se deberá usar coma (,) en el caso de las contribuciones redactadas en español y punto (.) en aquellas redactadas en inglés. 


\section{Lista de comprobación de preparación de envíos}

Como parte del proceso de envío, se les requiere a los autores que indiquen que su envío cumpla con todos los siguientes elementos:

1. El manuscrito no ha sido publicado previamente, ni se ha presentado a otra revista.

2. El archivo está en formato OpenOffice, Microsoft Word, RTF, o WordPerfect.

3. El texto tiene interlineado doble; el tamaño de fuente es 12 puntos; se usa cursiva en vez de subrayado y todas las tablas están al final del texto. Las figuras están en archivos aparte en los formatos apropiados.

4. El texto cumple con los requisitos bibliográficos y de estilo indicados en las instrucciones para los autores, que se pueden encontrar en Acerca de la revista. (Ver: http://intropica.unimagdalena.edu.co)

5. Se han añadido direcciones web para las referencias donde ha sido posible.

\section{Derechos de Autor}

\section{(c) (i) () (2)}

INTROPICA de la Universidad del Magdalena se encuentra bajo licencia Creative Commons 4.0 de Colombia: Reconocimiento-No Comercial-Compartir Igual. Cuando el autor cite el trabajo de otro o reproduzca una figura o una tabla de un libro o un artículo de revista, debe asegurarse que no está violando derechos de producción.

Aunque en general un autor puede reproducir tablas, fotografías u otras ilustraciones deberá obtener permiso del propietario de los derechos. Si el propietario de los derechos no es el autor del material citado o reproducido, se recomienda obtener también el permiso del autor. El material extraído de cartas y manuscritos no publicados no será aceptado a menos que se obtenga el permiso correspondiente. El autor siempre debe hacer un reconocimiento apropiado de cualquier material que le sea suministrado.

\section{Declaración de privacidad}

Los nombres y direcciones de correo-e introducidos en esta revista se usarán exclusivamente para los fines declarados por esta revista y no estarán disponibles para ningún otro propósito u otra persona. 


\section{GUIDE FOR AUTHORS}

INTROPICA is a scientific peer-reviewed journal published two times per year that provides a space for the publication of original and unpublished contributions that study and interpret, in an integrated way, tropical ecosystems and the environmental problems arising from the anthropogenic intervention on the territory.

INTROPICA is available for free on digital version (http://intropica.unimagdalena.edu.co) and in printed version (ISSN 1794-161X) and it is distributed to specialized libraries from academic, governmental and non-governmental institutions.

\section{Submission:}

Authors should register to submit manuscripts online in the website http://intropica.unimagdalena.edu.co or can submit their electronic manuscripts by email to revistaintropica@unimagdalena.edu.co. Receipt of manuscripts is permanent. The submission must include figures-graphs in separate files, and a letter of approval signed by all the authors certifying its originality and that it has not been submitted or been published elsewhere. The letter must indicate the type of contribution (Scientific article, Review article, Reflection article or scientific note) and two potential reviewers with the contact information. While all contribution are subject to prior review, the responsibility for its content lies with the authors and not on the editors, editorial advisory committee or the University of Magdalena. The format of the submission letter can be downloaded from the Journal website.

\section{Types of contributions:}

a) Scientific and technological research article. Corresponds to full results from studies on the topics addressed by the journal. Manuscripts should have a maximum of 30 pages, including tables and figures. The research paper should follow the following order: Title, Author (s), mailing address, Abstract and Keywords both in Spanish and English, Introduction, Materials and Methods, Results, Discussion, Conclusions (optional), Acknowledgements (optional), References, table(s) with relevant legend (s), list of figures legend. Figures should be submitted in separate files in a graphical format (tif, gif, eps).

b) Review article. Refers to critical summaries of recent insights in specific research areas that provide systematic and substantial coverage of subjects, and point out research lines to follow. The maximum length of the manuscript is 40 pages, including tables and figures. They must contain at least 50 references. Review articles can be written in a free style, however they should include Abstract, Keywords, Acknowledgements (optional), and References.

c) Reflection article. It presents the author's perspective, based on scientific literature, as a contribution to the knowledge on topics published by the journal. Manuscripts must have a maximum of 30 pages, including tables and figures. It must include Abstract and Key words in Spanish and in English, Acknowledgements (optional), and References.

d) Scientific note. Includes brief descriptions of limited research, experimental procedures, and technical or applied operations. In any case, they must be fully documented with reference to the literature, and indicating the experimental procedures used. This type of manuscript should have a maximum of 10 pages, including tables and figures. The Scientific Note should not have subtitles, but must include Abstract and Keywords both in Spanish and English, Acknowledgements (optional), and References.

e) Editorial. Document written by the editor, an editorial board member or a guest researcher in the thematic domain guidelines of the journal. Corresponds to a short document with few references. 


\section{Evaluation procedure/Peer review process}

The editorial board will make a first evaluation of the manuscript, conceptualizing its relevance and adequacy and it will be submitted for evaluation by at least two specialists in the field (double-blind review process). In case of controversy between the evaluators, a third peer will be resorted. These specialists will act as reviewers of the manuscript, they may make recommendations or request clarification and will have a maximum of one month to issue a concept on the manuscript. Subsequently, the suggestions of the editors and reviewers will be communicated to the authors, who have a one month to respond the suggestions. After that deadline, they will be treated as newly submitted manuscripts. The editors will decide whether to accept the manuscript for publication or not. The editors can be supported by the editorial advisory committee, when considered pertinent.

Prior to the final printing of manuscripts accepted for publication, the editors will send to the corresponding author (principal author) so that they perform the revision of galley proof, in order to make corrections for typing errors only. Major changes or additions to the edited manuscript are not allowed.

In case plagiarism is proved or the copyright violation, publishers will order the cancellation of the editorial process. For further information refer to the editorial best practice guidelines:

http://revistas.unimagdalena.edu.co/index.php/intropica/about/editorialPolicies\#custom-3

\section{Manuscript format:}

The text should be in Word or RTF format with a short name to identify the manuscript and the first author (eg Hifomicetos_López.doc). You must use double-spaced and the lines numbered through the text (including tables), Times New Roman font, size 12 and $2 \mathrm{~cm}$ margins on all four sides. Manuscripts in Spanish or English are accepted. All pages must be numbered consecutively. Tables are included in word format at the end of the manuscript (not paste them as pictures). Figures should be included in a separate document.

Scientific names and Latin phrases should be italicized. Do not include underlined words or indentation. In case of citation of a specific taxon or lower level, you must cite the scientific name including the author. Both authors and publishers shall be guided by the rules governing biological nomenclature, as agreed in the "International Code of Botanical Nomenclature," the "International Code of Nomenclature of Bacteria" and the "International Code of Zoological Nomenclature ". The author and year of publication of taxa are given only the first time the taxon is cited in the main text.

1. Title. Concise and informative. It must be provided in Spanish and English. Both the title of the manuscript and the titles of their main sections (rank one headings) should be capitalized and centrally placed. Rank two headings should be in lower-case and centrally placed. Ranked three should also be in lower-case but left-aligned. All titles and subtitles must be in boldface. In addition, the authors must provide an abbreviated title of maximum 80 characters including spaces.

2. Name (s) of the Author (s). Name each author in upper and lower case. For multi-authored papers, the main author's name should be placed at first.

3. Author (s) addresses. Provide the authors's affiliation, the full postal address of each affiliation, including phone numbers, and the e-mail address of the corresponding author. 
4. Abstract and "Resumen". The abstract must be written in Spanish (Summary) and English and should not exceed 300 words. The abstract should state briefly the objectives of the research, the experimental design (including sample sizes), the principal results and major conclusions that can be drawn from the findings. References should not be included in the abstract and this should be written in a single paragraph. The Abstract should be a faithful translation of the "Resumen".

5. Keywords. Immediately after the Summary and the Abstract, 3 to 6 keywords must be provided in Spanish and English, respectively, for indexing purposes.

6. Introduction. A revision of the updated references related to the work must be submitted (properly referenced sources), to support the hypothesis and show the objectives proposed.

7. Materials and Methods. The main materials must be included, in a clear and concise manner, as well as the methods used, so that another researcher can replicate the experiment. This section may be further divided into subsections, each with a concise subheading, as appropriate. Do not describe a method if it is already published; indicate the respective reference in that case and only relevant modifications should be described.

8. Results. This section should be clearly and concisely presented, including tables, figures, and statistical analysis, if necessary.

9. Discussion. The results should be contrasted with the background knowledge, highlighting the contribution of the article to the understanding of the subject addressed. The conclusions drawn from the work should be consistent and related to the results presented. A combined Results and Discussion section can be presented.

10. Acknowledgements. If deemed necessary by the authors, appreciation for financial contributions, advisories, donations or another type of collaboration relevant to the work may be included.

11. References. Any references cited in the text must be given in full and should be in alphabetical order according to the first author's last name. Authors are responsible for the accuracy of references. INTROPICA follows the Harvard style citation system, so that we encourage you to consider the following guidelines:

- The manuscript should be carefully checked to ensure that the spelling of authors' names and years are exactly the same in the text as in the reference list.

- The references cited in the text should refer to the name(s) of author(s) and publication year. For example, "Rodriguez (1998) found that ..." "A multinomial technique is described for estimating the variance associated with proportions (Kent and Lane, 2004)".

- If a work has more than two authors, cite the first author's name only, followed by "et al." without italics. In the reference list, the names of all authors must be mentioned.

- More than one reference from the same author(s) in the same year must be identified by consecutive letters placed after the respective year of publication If there are several works by the author (s) in the same year, it will be cite with a letter to identify the respective year (example: García et al., 1998a, b, c).

- Citations in the same sentence should be arranged chronologically. For example, "Biological indicators are widely used for environmental analysis (Platt, 1964; Arias and Pérez, 1980; Klimm et al., 2000).”

- The scientific journal titles should be complete, not abbreviated and italicize

- The title of the references must retain the original language.

- "Unpublished results" and "personal communication" are not recommended in the reference list, but may be mentioned in the text 
Use the following format in the References section:

- Periodical publications or journals:

Pérez, R., Condit, R., Aguilar, S., Hernández, A. and Villareal, A. 1996. Inventario de la vegetación de la isla de Coiba, Panamá: Composición Florística. Revista de Biología Tropical 44: 31-40.

Cubides-Guerrero, P.A. and Ramírez-Franco, J.H. 2014. Adsorción de Cr VI sobre residuos de café. Revista Mutis 4(2): 18-25 http://revistas.utadeo.edu.co/index.php/mutis/article/view/953/992. Accesed January 2, 2015

- Books

Longhurst, A. and Pauly, D. 1987. Ecology of tropical oceans. Academic Press, San Diego.

- Book chapters:

Bucker, D. 1989. Histology. En: Austri, D., Editor. Methods for the Microbiological Examination of Fish and Shellfish. Wiley, New York.

- Thesis

Gil, A. 2000. Evolución bioquímica de los endosimbiontes en insectos asociados con el maíz en el sur de México. Doctoral thesis, Universidad Agrícola, Mérida, Yucatán, México.

- Websites

Alexander, J. and M.A. Tate. 2001. Evaluando las Fuentes Electrónicas. Widener University._URL:_http://www2. widener.edu/Wolfgram-MemorialLibrary/ webevaluation/ webeval.htm. Accesed October 9, 2012.

\section{Tables}

- Do not embed "graphically designed" tables, but prepare these using the wordprocessor's facility.

- Tables should be numbered according to their sequence in the text with Arabic numerals including a brief and descriptive caption at the top. It is recommended to use clear and informative table captions, avoiding consulting the text to understand the table.

- Each table should appear on a separate page at the end of the text and before the figure legends.

- Information showed in the tables should not appear repeatedly in the figures.

- The column headings should be brief but explanatory.

- Do not use bold fonts.

- Write in parentheses the standard abbreviations of measurement units.

- Do not use vertical lines to separate columns; instead, include extra spaces between them.

- Any additional explanation for the understanding of the table should be supplied as a table footnote.

\section{Figure captions}

- Include at the end of the text, in a separate page, a list with the figure captions.

- Do not use bold fonts.

- It is recommended that the legends be informative, so the reader does not need to search in the text what the figure shows.

- It is recommended that required explanations be written in the legend to prevent text inside the figures. 


\section{Figures}

- Figures (drawings, maps, films, photographs and computer graphics) should be sent in separate files, graphic high quality digital format (tiff, bmp, jpg or gif), with a minimum resolution of 300 dpi. If possible, we recommend sending vectorial graphics files.

- Figure files should be named using the first author name followed by the figure number. Example: López fig.1.jpg.

- Figures shall be numbered with Arabic numerals according to their sequence of appearance in the text.

- The original size of the figure should allow a reduction of $50 \%$ without loss of clarity.

- Composite figures with multiple images are listed consecutively. Example: Figure 1a, 1b, 1c, etc.

- Use the same kind of letter for all the illustrations and follow the style of the journal.

\section{Units}

The measures must be expressed in units of the international system, a space between the number and the unit of measurement is allowed and must not use full stop following the abbreviation $\left(15 \mathrm{~m}, 10 \%, 20{ }^{\circ} \mathrm{C}, 7 \mathrm{~kg}\right)$. The abbreviation of units of measurements should be used only if they are preceded by numbers. Salinity values should be expressed without units or symbols.

\section{Formulas}

- Mathematical equation must be written as a editable text and not as an images

- Provide the meaning of all symbols immediately after the equation in which they are first used.

- For simple fraction used the symbol "/" instead of the horizontal line, Example: $S y / X m$ instead of $\frac{S y}{X m}$

- Equations referenced in the text must be numbered on the right side in parenthesis.

- The statistical significance must be indicated with the letter "p" in lowercase and without no space between the sing and the value of significance

\section{Decimals}

The authors must use a comma (,) in the case of contribution written in Spanish and dot (.) when the manuscript is submitted in English.

\section{Submission preparation Checklist}

As part of the submission process, authors are required to check the following items:

1. The manuscript has not been previously published, nor has been submitted to another journal.

2. The file is sent in Open Office, Microsoft Word, RTF, or WordPerfect format.

3. The text is double-spaced; the font size is 12 points; employs italics, rather than underlining and all tables are at the end of the text. Figures are in separate files in the appropriate formats.

4. The text follows to the style and bibliographic requirements outlined in the Author Guidelines which can be found in the Journal webpage. (See: http://intropica.unimagdalena.edu.co).

5. Web addresses have been added for the references if possible. 


\section{Copyright Notice}

\section{(c) (i) (9)}

INTROPICA by Universidad del Magdalena is licensed under a Creative Commons Reconocimiento-NoComercial-CompartirIgual 4.0 Internacional License. When the author cites the work of another author or uses a figure or table from a book or journal article, he/she must make sure that they are not violating production rights (Copyright).

Although in general an author may reproduce tables, photographs or other illustrations must obtain permission from the copyright holder. If the copyright owner is not the author of the quoted or reproduced material, it is also recommended to obtain permission from the author. The material extracted from unpublished letters and manuscripts will not be accepted unless a permit is obtained. The author should always make a proper acknowledgment of any material that will be provided to him/her.

\section{Privacy Statement}

The names and email addresses entered in this journal will be used exclusively for the stated purposes of this journal and will not be available for any other purpose or used by another person. 


\section{Índice periódico por volumen}

\section{Volumen $13 \mathrm{~N}^{\circ} 1$ enero- junio de 2018}

\section{Editorial}

Retos del milenio: la pobreza extrema y el hambre, una reflexión

Challenges of the new millenium: a reflection on extreme poverty and hunger

Pedro Eslava-Eljaiek

\section{Artículos de investigación científica y tecnológica}

Effects of experimental pool level reduction on phylloicus pulchrus (trichoptera: calamoceratidae) feeding and conspecific behavior from a tropical rainforest stream Efecto de reducción experimental de pozas sobre la alimentación y comportamiento conespecífico de Phylloicus pulchrus (Trichoptera: Calamoceratidae) de una quebrada de bosque tropical lluvioso

Limarie J. Reyes-Torres and Alonso Ramírez

Eficiencia y selectividad de carnada y anzuelo en el uso del palangre para la captura del pez "cabeza de tornillo" espina larga (Sebastolobus altivelis) en aguas profundas de Baja California, México

Efficiency and selectivity of bait and hooks in the use of the longline the catch of tornyhead long spine (Sebastolobus altivelis) in deep waters of Baja California, México

Jorge Flores Olivares, Alfredo Emmanuel Vázquez Olivares y Antonio Murillo Olmeda

Caracterización morfométrica de una laguna costera neotropical (Ciénaga el Chino, Magdalena - Colombia)

Neotropical coastal lagoon's morphometric characterization (Ciénaga El Chino, Magdalena Colombia)

Jaime de Jesús Rodríguez Curcio, Jean Linero-Cueto y Leandro Jose Barros Ortíz

Macroinvertebrados bentónicos como indicadores de calidad de agua en la cuenca del Pachanlica, Provincia DE Tungurahua, Ecuador

Benthic macroinvertebrates as indicators of water quality in the Pachanlica Basin, Tungurahua Province, Ecuador

Marcia Buenaño, Carlos Vásquez, Hernán Zurita-Vásquez, Giovana Parra y Ruth Pérez 


\section{Notas Científicas}

Compuestos nitrogenados en los sedimentos del Estero San Camilo: Guayas, Ecuador y su relación con actividades antrópicas adyacentes

Nitrogen compounds in the sediments of the San Camilo mat: Guayas, Ecuador and its relationship with adjacent anthropic activities

Daniela Limongi, Maritza Cárdenas-Calle, Luis Troccoli y Juan Carlos Erazo

Diagnóstico clínico de monogeneos en alevinos de piscicultura intensiva en Arauca Clinical diagnosis of monogenea in fingerling fish farm in Arauca

Daniel Leonardo Cala Delgado, Norquis Caled Álvarez Rubio, Fabián Andrés Muñoz Rodríguez,

Carlos Alberto Blanco Torres y Jefferson Yunis Aguinaga 


\section{Índice periódico por volumen}

\section{Volumen $13 \mathrm{~N}^{\circ} 2$ julio - diciembre de 2018}

\section{Artículos de investigación científica y tecnológica}

Diversidad y estructura genética del Prochilodus magdalenae (Pices: Prochilodontidae) aguas arriba y abajo de la represa Betania, Colombia

Diversity and genetic structure of Prochilodus magdalenae (Pisces: Prochilodontidae) upstream and downstream Betania dam, Colombia

Paulin P. Fontalvo, Gilberto Orozco-Berdugo y Juan Narváez-Barandica

Calidad del agua de la parte baja del río Córdoba (Magdalena, Colombia), usando el ICA-NSF

Water quality in the lower section of Córdoba River (Magdalena, Colombia), using the ICA-NSF

Fernando Antonio Fontalvo-Julio y Cesar E. Tamaris-Turizo

Estructura del ensamblaje de macroinvertebrados bentónicos de una laguna urbana en la Pampa Mesopotámica

Structure of the benthonic macroinvertebrate assemblage of an urban lagoon in the Mesopotamic Pampa

Melina Crettaz-Minaglia, Diamela Gianello, Carlos Roldán, Eduardo Chaves, Irene Aguer y

Ricardo A. Juárez

Insectos depredadores y parasitoides asociados al cultivo de soya (Glycine max L. Merrill, 1917) en el Distrito Arquitecto Tomás Romero Pereira, Itapúa, Paraguay

Predator and parasitoid insects associated with soybean crop (Glycine max L. Merrill 1917), in the District Arquitecto Tomás Romero Pereira, Itapúa, Paraguay

Erika Viviana Stepin-Sytni, María Cristina Garay-Villalba, Marco Maidan- Ojeda y Claudia

Cabral-Antúnez

Parasitismo natural de Spodoptera frugiperda (Smith) (Lepidoptera: Noctuidae), en cuatro departamentos de Paraguay

Natural parasitism of Spodoptera frugiperda (Smith) (Lepidoptera: Noctuidae) in four departments in Paraguay

Claudia C. Cabral-Antúnez, Bolívar Garcete, Rocío I. Montiel-Cáceres, Alexis B. Gonzalez-Vega,

Sergio R. Cárdenas, Nancy Armoa y María Bernarda Ramírez de López 


\section{Artículos de revisión}

Biocomercio y sostenibilidad: análisis en torno a las materias primas de las artesanías en Colombia

Biocomerce and sustainabillity: an analysis around raw materials in Colombian handicrafts

Luisa Fernanda Casas-Caro y Alejandro Lozano

Apuntes sobre la importancia ecológica, ambiental y social de la arenca Triportheus magdalenae (Steindachner, 1878). Un ejemplo de endemismo invisibilizado

Notes about ecological, environmental and social importance of the arenca Triportheus magdalenae. An example of invisibilized endemism

Juan Carlos Valdelamar-Villegas

\section{Artículos de reflexión}

El impacto ambiental de los residuos de promoción electoral en Ecuador: caso de estudio de las lonas publicitarias

Environmental impact of electoral campaigns waste in Ecuador: advertising banners case

Hellen Arichábala-Martínez 